\title{
Size and Shape of TBM Debris Estimated by the Nishimatsu's Cutting- Resistance Equation
}

\author{
S. Okubo, K. Fukui* and W. Chen
}

Department of Systems Innovation, The University of Tokyo, Tokyo, Japan

\begin{abstract}
Information concerning the size and shape of tunnel boring machine (TBM) debris is essential for designing efficient loading, hauling and dumping systems for TBM excavation. It is also very important in deciding how to recycle the TBM debris. However, only very limited information is available at present, and theoretical or calculation methods to estimate the size and shape are still open to discussion. In this paper, the size and shape of TBM debris are estimated by the well-known Nishimatsu's equation that is usually applied to roadheaders or shield-type machines with chisel bits. In this study, the equation was applied to the case of TBM excavation with disc cutters in which the shear failure or plane extends from a new groove to an adjacent pre-existing one. The estimated size and shape of TBM debris were found to be consistent with the measured results. Side forces applied to a disc cutter and the resultant stress on the cutter were considered. The maximum debris size encountered in tunnel excavation was also discussed assuming that it followed the Gumbel distribution. The results suggest that the proposed approach based on Nishimatsu's equation shows potential for future study.
\end{abstract}

Keywords: TBM, Debris, Size, Shape, Nishimatsu's equation, Gumbel distribution.

\section{INTRODUCTION}

The debris expelled by a tunnel boring machine (TBM) varies widely in size and shape under the influences of many factors [1]. Observation of the size and shape of the debris allows one to judge whether excavation is proceeding smoothly and what the rock mass conditions are. It is well known that the larger the debris, the greater the efficiency of the excavation $[2,3]$. Debris size and shape become criteria for whether or not it can be reused; for example, as the aggregate in concrete $[4,5]$. However, knowledge about the size and shape of debris is generally passed down at individual work sites, and little basic research has been conducted. Finite element models (FEM) and other numerical calculations have become made remarkably more sophisticated in recent years and promise to be useful for basic research on debris [6], but some analytical investigations will first be necessary to form a basic understanding of the problem. In the present study we investigate the size and shape of debris using Nishimatsu's expression for cutting resistance (hereinafter, Nishimatsu's equation) [7], which is the most widely known expression among those dealing with rock cutting.

\section{MERCHANT'S EQUATION AND NISHIMATSU'S EQUATION}

It is well known that Nishimatsu proposed his equation for the cutting resistance of a cutting tool on the basis of Merchant's equation [8, 9]. In contrast to Merchant's emphasis of the cutting of metals, Nishimatsu mainly focused on

*Address correspondence to this author at the Department of Systems Innovation, The University of Tokyo, Tokyo, Japan; Tel: +81 358417065 ; Fax: +81 35841 7035; E-mail: fukui@sys.t.u-tokyo.ac.jp cutting rock, but the two have much in common. Therefore, this paper will also employ Merchant's equation.

Fig. (1) is a conceptual diagram of orthogonal cutting with a cutting tool. The depth is assumed to be 1 unit [7]. Here, $\alpha$ is the rake angle measured in the clockwise direction from the vertical axis. The value of $\alpha$ is often negative in cutting tools for hard rock in which the edge angle is large in order to prevent chipping. In the figure, the cutting tool is assumed to move from right to left as it cuts the rock, at which time $F$ is the resultant force on the rake face. $\phi$ is the angle $F$ makes with a vector normal to the rake face and is measured in the counter-clockwise direction.

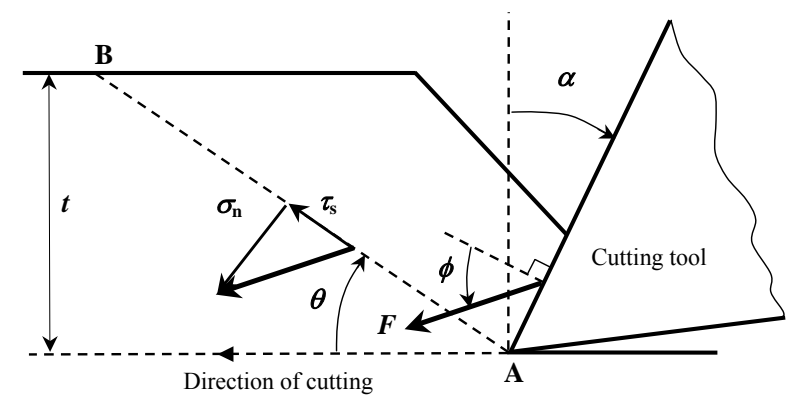

Fig. (1). Stress distribution and cutting forces for orthogonal cutting [5].

Both Merchant and Nishimatsu assumed that fracture occurs on the shear plane (AB) in Fig. (1). In Merchant's equation, the resultant stress $p$ on the shear plane is assumed to be uniform. Nishimatsu assumed, however, that $p$ reached a maximum value at point $\mathrm{A}$ and then diminished monotonically at points closer to $\mathrm{B}$. He approximated this change with the following equation: 


$$
p=p_{0}\left(\frac{t}{\sin \theta}-\lambda\right)^{n}
$$

where $t$ is the cutting depth, $\theta$ is the angle between the horizontal axis and the shear plane $\mathrm{AB}, \lambda$ is the distance measured along the shear plane from the origin $\mathrm{A}$, and $n$ is a constant determining the distribution of the resultant force distribution. $p_{0}$ is determined by force equilibrium and is proportional to $F$. The above assumptions are the largest points of difference between Nishimatsu's and Merchant's approaches.

The parameter in Merchant's and Nishimatsu's equations that is most difficult to determine and therefore the most arbitrary is $\theta$ in Fig. (1). The following are the three typical approaches for determining $\theta[10]$ :

1. Krystof's Assumption: A simple and clear proposal, this assumes that the shear plane occurs at a $45^{\circ}$ slope relative to the resultant cutting force $F$. This assumption considers the shear planes occurring in a material with a small internal friction angle during a uniaxial compression test.

2. Merchant's First Assumption: It is assumed that the shear plane occurs such that the energy is minimized. Specifically, the value of $\theta$ that minimizes the horizontal component of $F$ is employed.

3. Merchant's Second Assumption: The shear strength $\tau_{\mathrm{S}}$ varies with the stress $\sigma_{\mathrm{n}}$ normal to the shear plane. $\theta$ is then calculated as stated in Merchant's First Assumption.

$\pm \tau_{S}=S_{S}-\sigma_{n} \tan k$

$\mathrm{S}_{\mathrm{S}}$ stands for the shear strength when $\sigma_{\mathrm{n}}=0$ and corresponds to cohesion in Coulomb's well-known failure criterion. $k$ is the internal friction angle. Nishimatsu [7] also made this assumption.

Researchers have carried out numerous investigations of the above assumptions $[7,10]$. There are various approaches to evaluating these assumptions, but one of the most powerful is to draw a graph with $\theta$ on the vertical axis and $\alpha-\phi$ on the horizontal axis. In this graph, $\theta$ based on Krystof's assumption then becomes the lower limit, while Merchant's First Assumption becomes the upper limit. Under Merchant's Second Assumption, $\theta$ lies between the two limits. Considering this, Nishimatsu adopted the Merchant's Second Assumption.

Let us summarize the above statements before proceeding with the investigation based on Nishimatsu's equation.

1. Nishimatsu assumes that the shear strength varies with the stress normal to the shear plane. This is the same as Merchant's Second Assumption.

2. Merchant assumes that the resultant force on the shear plane (AB) in Fig. (1) is uniform, but Nishimatsu assumes that it follows the distribution given by Eq. (1). This is the main difference between the two.

3. Equations (6) - (8) from reference [7], which represent Nishimatsu's final result, are identical to those of Merchant, except for the values of the constants.

\section{APPLICATIONS TO DISC CUTTER (TBM)}

\section{(When There is no Neighboring, Pre-Existing Groove)}

Nishimatsu's equation approximates the resultant force $F$ at the rake face using the following equation, which has 6 parameters on the right side:

$$
F=\frac{2}{n+1} S_{S} t \frac{\cos k}{1-\sin (k-\alpha+\phi)}
$$

When the TBM is a disc cutter type as in Fig. (2), F corresponds to the so-called "side forces" arising at the sides of the cutters. The tip of the disc cutter in recent TBMs is tabular and the rake angle has a low absolute value; therefore, a similar shape was assumed in this study (Fig. 2).

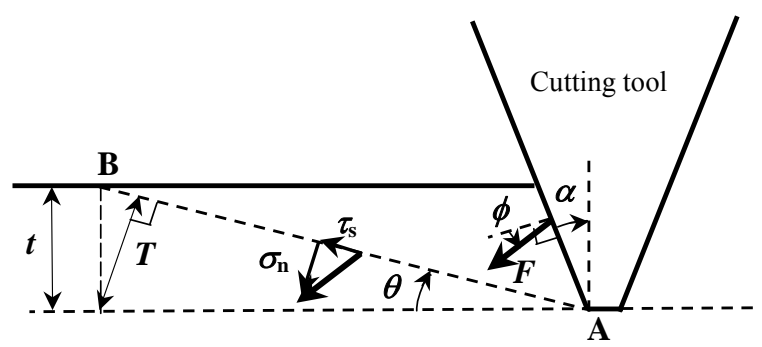

Fig. (2). Stress distribution and cutting forces for case of disc cutter. In this figure, $\alpha$ takes a negative value (negative rake angle).

$\theta$ is the parameter controlling the shape of debris when a cutter is making an initial cut in the local rock surface, with no pre-existing, adjacent groove. The greater $\theta$ is, the lower the length:thickness $(L / T)$ ratio is in the ejected debris; conversely, low $\theta$ results in the ejection of flat debris with large $L / T$ ratios. Using Nishimatsu's equation, $\theta$ can be expressed as follows [7]:

$\theta=45-\frac{k}{2}-\frac{\phi-\alpha}{2}$

The variation in $\theta$ with $\phi-\alpha$ was drawn in Fig. (3).

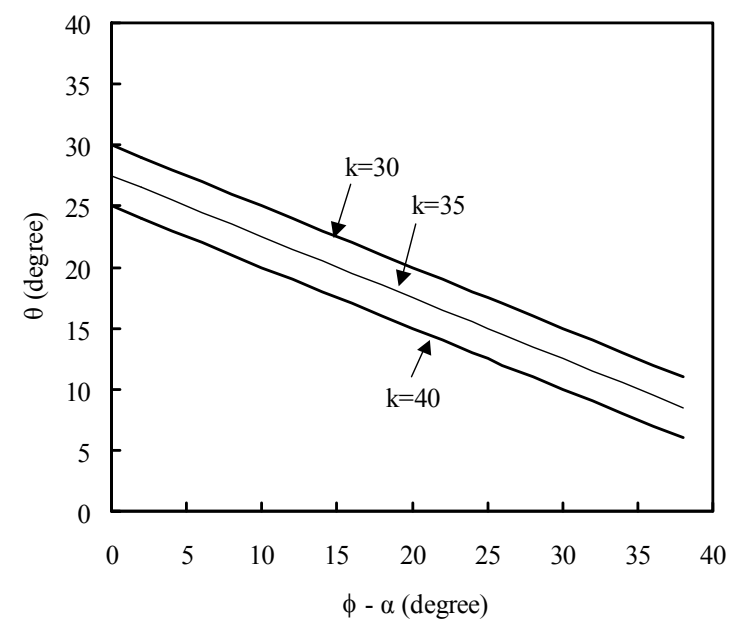

Fig. (3). Angle between the direction of cutting and the shear plane, $\theta . \alpha$ is the rake angle of the cutting tool, and $\phi$ is the angle between the direction of the resultant cutting force and the normal to the rake face of the cutting tool, termed 'the angle of friction of rock cutting'. $k$ is the internal friction angle of the rock sample. 
The length, breadth and thickness of a piece of debris are written $L, B$ and $T(L>B>T)$. The values of $L$ and $T$ in the debris in Fig. (2) are assumed as follows:

\section{$L$ : Length of base of triangle (AB)}

\section{$T$ : Length of vertical line from base $(\mathrm{AB})$ to vertex}

There are various methods for determining $L$ and $T$, but when the absolute value of $\alpha$ is low, the value of $L / T$ does not show any large variation among the assumptions. Using the above definitions to find $L / T$, a simple calculation reveals it as $1 /\{\sin (\theta) \cdot \cos (\theta)\}$. Fig. (4) shows the relation between $L / T$ and $\phi-\alpha$. When $\phi=0$ and $\alpha=0$, a force acts in the horizontal direction and $\theta$ is dependent on the internal friction angle $k$, as in the case of the uniaxial compression test. As $\phi$ increases, $\theta$ decreases and a downward component of force appears. As $\alpha$ decreases, $\theta$ decreases. The value of $\alpha$ has become negative in recent chisel bits for use in hard rock and the TBM disc cutters discussed herein, so it is expected that $\theta$ will become quite small.

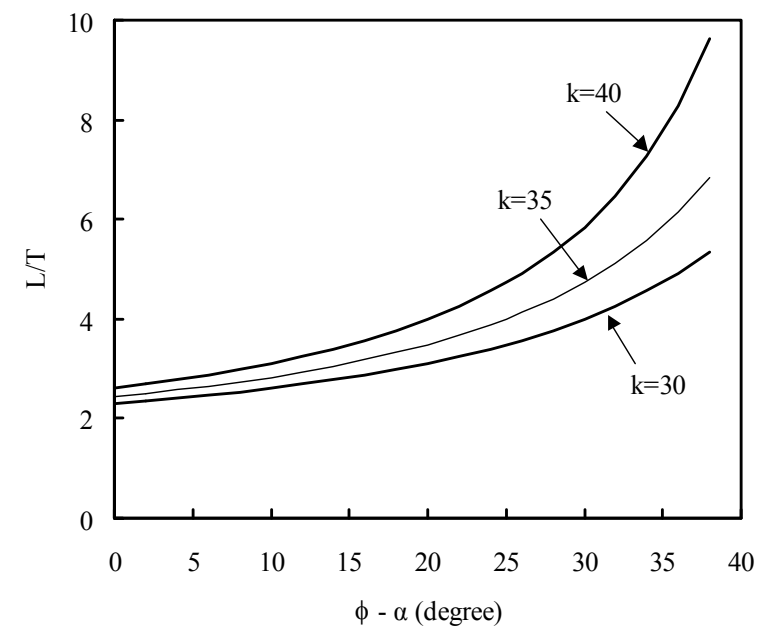

Fig. (4). Debris length normalized by debris thickness, $L / T$.

The parameter values are investigated in the discussion below. From Fig. (5) in Nishimatsu's publication [7], $\phi$ is set to $24^{\circ}$ (using the $y$-intercept). Then, the frictional coefficient on the rake face, $\tan \phi$, is quite high at 0.45 , but that can be attributed to the fact that the frictional coefficients in Nishimatsu's and Merchant's equations include cutting resistance in the primary crushed zone at the cutting tool tip.

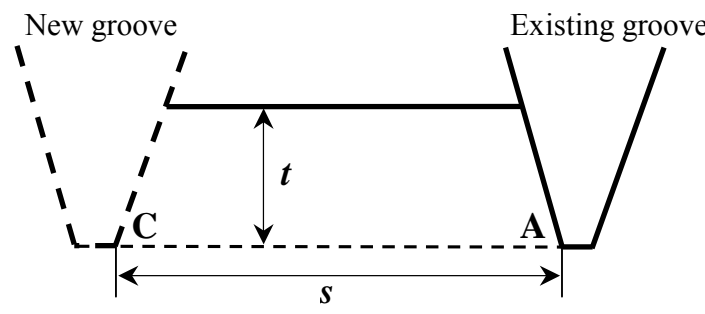

Fig. (5). Extension of a shear plane from the cutting tool tip to an existing groove.

$\alpha$ is assumed to lie in the range $-5^{\circ}$ to $0^{\circ}$.

$k$ is assumed to lie in the range $30^{\circ}$ to $40^{\circ}$.

Then, $\phi-\alpha$ varies in the range $24^{\circ}-29^{\circ}$. Additionally, when $\phi-\alpha=26^{\circ}, L / T$ takes on the following values:

$\begin{array}{lll}k=30^{\circ} & \theta=17^{\circ} & L / T=3.6 \\ k=35^{\circ} & \theta=14.5^{\circ} & L / T=4.1 \\ k=40^{\circ} & \theta=12^{\circ} & L / T=4.9\end{array}$

Within the range given in Fig. (4), the minimum magnitude of $L / T$ is 2.3 and the maximum is 9.6. Debris from TBM, the object of interest here, commonly has $L / T$ in the range $2-10$. These will be treated in more detail in part 4 , but Table 1 shows the number of particles, uniaxial compressive strengths and dimensions of debris in samples taken in Takisato Tunnel. The values for any of these parameters have some arbitrariness, but after a comparison of the computation results in Fig. (4) with the measurements of the shape of debris provided in Table $\mathbf{1}$, it can be seen that there is no great contradiction between the two.

\section{(When There is an Adjacent Groove)}

Let us consider the case when there is an adjacent, preexisting groove, as shown in Fig. (5) [11]. To touch briefly on excavation efficiency, the energy efficiency is high if a single pass (a single rotation of the cutter head) is sufficient to establish coupling between neighboring grooves; in a multiple pass situation, much energy is wasted for multiple rotations. As in Fig. (1), the object in Fig. (5) is assumed to have unit depth. The step distance to the adjacent groove is $s$. It is assumed that coupling occurs between the existing groove and the new groove, i.e., that a shear plane occurs from origin A to point $\mathrm{C}$. The shear plane between the coupled grooves is not necessarily approximately planar [12],

Table 1. Sampling Locations in Takisato Tunnel and Measured Data of Collected Debris.

\begin{tabular}{|c|c|c|c|c|c|c|c|c|c|}
\hline Tunnel Distance (m) & 1860 & 2115 & 2280 & 2625 & 2660 & 2680 & $\mathbf{A}$ & B & $\mathbf{C}$ \\
\hline Number of debris & 252 & 706 & 499 & 259 & 698 & 302 & 260 & 364 & 238 \\
\hline Uniaxial compressive strength (MPa) & 70 & 42 & 42 & 47 & 50 & 58 & - & - & - \\
\hline$L(\mathrm{~cm})$ & 4.38 & 4.26 & 3.82 & 3.98 & 4.06 & 4.03 & 4.23 & 3.98 & 4.27 \\
\hline$B(\mathrm{~cm})$ & 2.74 & 2.63 & 2.49 & 2.56 & 2.62 & 2.55 & 2.71 & 2.70 & 2.88 \\
\hline$T(\mathrm{~cm})$ & 1.28 & 1.22 & 1.53 & 1.30 & 1.51 & 1.27 & 1.25 & 1.52 & 1.36 \\
\hline$L / T$ & 3.67 & 3.95 & 2.72 & 3.32 & 2.92 & 3.46 & 3.73 & 2.86 & 3.46 \\
\hline$B / T$ & 2.29 & 2.45 & 1.76 & 2.15 & 1.88 & 2.20 & 2.38 & 1.94 & 2.32 \\
\hline
\end{tabular}


but here, we follow that assumption of Merchant's and Nishimatsu's equations in our analysis.

If, in order to preserve compatibility with Eq. (1), we assume that the distribution of the resultant force on line AC obeys a power law, we obtain the following expression:

$$
p=p_{0}(s-\lambda)^{n}
$$

Here $\lambda$ is the distance from origin A toward C. The integral of $p$ from $\mathrm{A}$ to $\mathrm{C}$ must be in equilibrium with the resultant force $F$, so we can set the integration of Eq. (5) from $\lambda=$ 0 to $s$ equal to $F$, obtaining:

$$
F=-\frac{1}{n+1} p_{0} s^{n+1}
$$

Eliminating $p_{0}$ from Eqs. (5) and (6), we obtain

$$
p=-(n+1) F \frac{(s-\lambda)^{n}}{s^{n+1}}
$$

We then obtain the value at point A by setting $\lambda=0$ in Eq. (7)

$$
p_{A}=-(n+1) \frac{F}{s}
$$

Separating $p_{\mathrm{A}}$ into normal and shear stresses, we obtain the following:

$$
\begin{gathered}
\sigma_{A}=-(n+1) \frac{F}{S} \sin (-\alpha+\phi) \\
\tau_{A}=-(n+1) \frac{F}{S} \cos (-\alpha+\phi)
\end{gathered}
$$

We substitute the above 2 equations into Coulomb's failure criterion in Eq. (2) to obtain the following expression.

$$
S_{S}=-(n+1) \frac{F}{S}\{\cos (-\alpha+\phi)-\tan k \sin (-\alpha+\phi)\}
$$

Substituting Eq. (3) into this and rearranging, we find

$$
\frac{s}{t}=\frac{2 \cos (k-\alpha+\phi)}{1-\sin (k-\alpha+\phi)}
$$

Thus, the condition for coupling to occur on $\mathrm{AC}$, rather than on $\mathrm{AB}$, can be written as follows:

$\frac{s}{t}<\frac{2 \cos (k-\alpha+\phi)}{1-\sin (k-\alpha+\phi)}$

Fig. (6) was drawn after these conditions had been calculated. If we assume, as before, that $\phi-\alpha=26^{\circ}$, then we obtain the following expressions:

$$
\begin{array}{ll}
k=30^{\circ} & s / t<6.5 \\
k=35^{\circ} & s / t<7.7 \\
k=40^{\circ} & s / t<9.4
\end{array}
$$

\section{INVESTIGATION USING DEBRIS COLLECTED IN-SITU}

\section{(Collection of Debris and Debris Shape)}

An investigation was conducted on the debris collected in the Takisato water conveyance tunnel (Takisato tunnel) [2], which was excavated with an 830-cm diameter TBM. The diameter of the disc cutter in the TBM used for the Takisato tunnel was $43.2 \mathrm{~cm}$, the distance (spacing) between the disc cutters (distance between adjacent grooves) was $8 \mathrm{~cm}$, and the mean cutting depth $t$ was about $1 \mathrm{~cm}$. Debris was compressed up to failure between two flat platens and the peak load was converted to uniaxial compressive strength [13]. The rock mass was comparatively hard sandstone and mudstone, with a uniaxial compressive strength of $45-100 \mathrm{MPa}$. The debris was carried $100 \mathrm{~m}$ from the tunnel face on a conveyor belt and then transported by tram to a temporary disposal site outside the tunnel. Samples of about $20 \mathrm{~kg}$ each of debris were taken 6 times from the temporary disposal site. In addition, soft debris was also collected from 3 disposal locations designated $\mathrm{A}, \mathrm{B}$ and $\mathrm{C}$ which were used to dispose of debris from initial excavation. The reader is directed to a previous report [2] for descriptions of the particle size distributions in the debris.

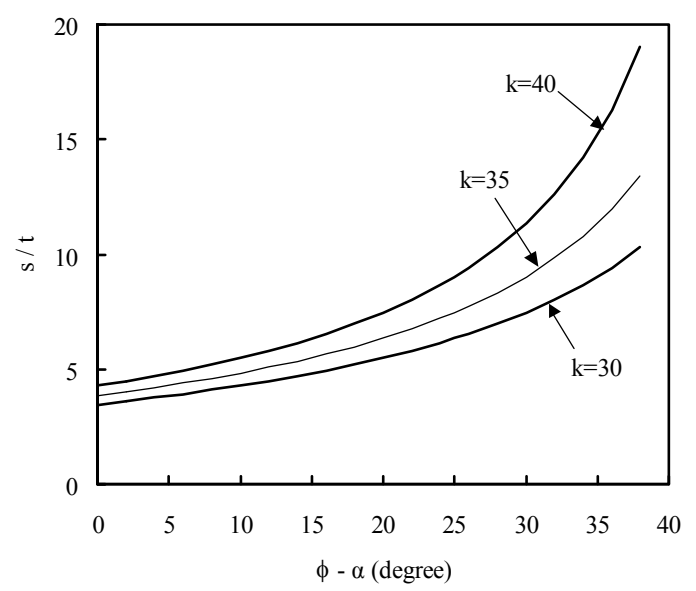

Fig. (6). Critical distance (spacing) between adjacent grooves, $s$, normalized by cutting depth, $t$.

Debris particles weighing at least $5 \mathrm{~g}$ were sorted and their dimensions $L, B$ and $T$ were measured. The figures given in Table 1 indicate the numbers of such particles, the uniaxial compressive strength, and the particle shapes. The mean length $L$ was $4.11 \mathrm{~cm}$, roughly half the value of $s$. The reader can see in the table that the proportion $L: B: T$ was roughly $3: 2: 1$. The values for these variables and for $L / T$ and $B / T$ showed considerable scatter; the coefficients of variance for these were $0.4-0.44$. The interesting aspect here is that, as shown in Fig. (7), $L / T$ and $B / T$ remain approximately constant in spite of variations in $T$. This is a fascinating trend and the authors intend to collect more data related to this from other locations.

\section{(Comparison with Computation Results Based on Nishimatsu's Equation)}

If we summarize our results using Nishimatsu's equation, we find that the shear plane occurs at angle $\theta$ while $T$ (or $t$ ) is small; once $T$ exceeds a certain value, however, coupling occurs with the adjacent groove. Fig. (8) shows the theoretical curves occurring when $k=30^{\circ}, 35^{\circ}$ and $40^{\circ} . \phi-\alpha=26^{\circ}$ was assumed for the calculations, as before. Initially, the slope is the same as when there is no adjacent groove. Cou- 
pling with the adjacent groove occurs as soon as Eq. (8) is satisfied, resulting in a jump in the data on the graph. $L$ changes to $\mathrm{s}$ and does not change further even if the cutting depth is increased. The debris thickness changes from $T$ to the cutting depth $t=T / \cos \theta$. The data from Takisato Tunnel are graphed on the same figure. The points indicating the mean values for the data are shown on the large circle. We can say the following from this figure:

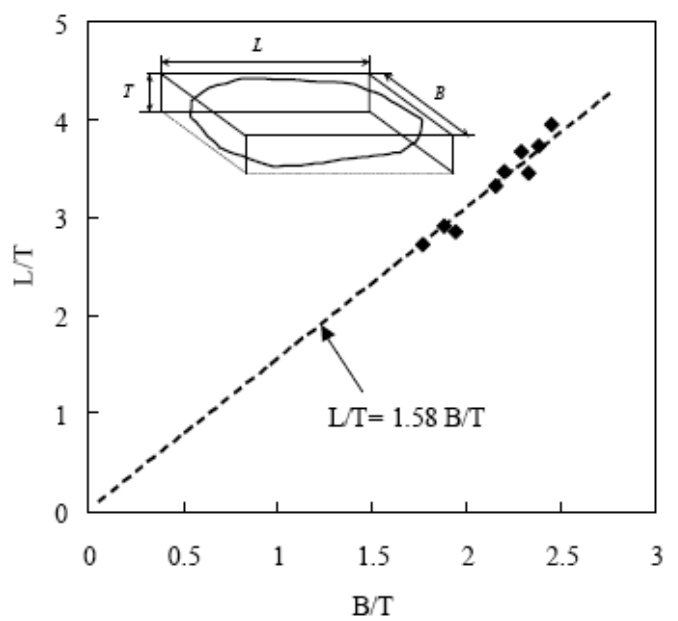

Fig. (7). Measured debris length, $L$, and width, $B$, normalized by debris thickness, $T$.

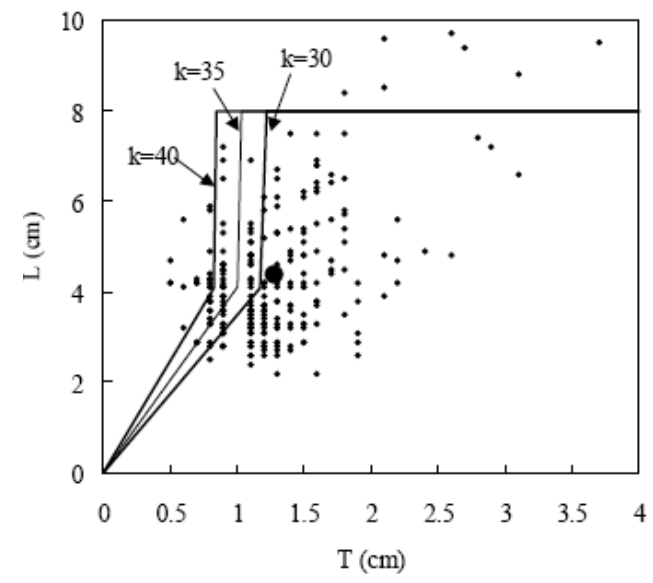

Fig. (8). Measured debris length, $L$, and debris thickness, $T$, in Takisato tunnel.

1. We collected debris that weighs at least $5 \mathrm{~g}$. However, debris in this size range includes most of the debris that is addressed by Nishimatsu's equation. This suggests that smaller debris is generated collaterally with large debris or derived from secondary fracturing.

2. The mean cutting depth was about $1 \mathrm{~cm}$ in this tunnel. In view of this, the large red circle representing mean values is plausibly positioned.

3. The slip face is not necessarily a simple shear plane, but may take an arched shape, so $T$ may be larger than the cutting depth.

4. There is debris with large $L$ in the direction of the cutter movement.

\section{DISCUSSION}

\section{(Forces Acting on the Disc Cutter and Resultant Forces)}

There have been many analyses of the rolling force of the disc cutter and the thrust force $[14,15]$, but an inadequate number of studies of the axial-direction forces on the disc cutters. Ordinarily, one assumes, identical forces act in equilibrium on the left and right side of the disc cutter; however, when there are adjacent grooves and cracks are forming in the rock between any cutter and an adjacent groove, a force is only exerted at the cutter surface opposite the adjacent groove. Let us estimate the force $P$ in the lateral direction when Eq. (8) is exactly satisfied. The procedure is to calculate $F$ using Eq. (3), and then $P$, using the following expression [7]:

$P=F \cos (\varphi-\alpha)$

The next numerical values are assumed, and the results are shown in Fig. (9).

$$
\begin{aligned}
& n=11 \\
& S_{\mathrm{s}}=40 \mathrm{MPa} \\
& t=1 \mathrm{~cm}
\end{aligned}
$$

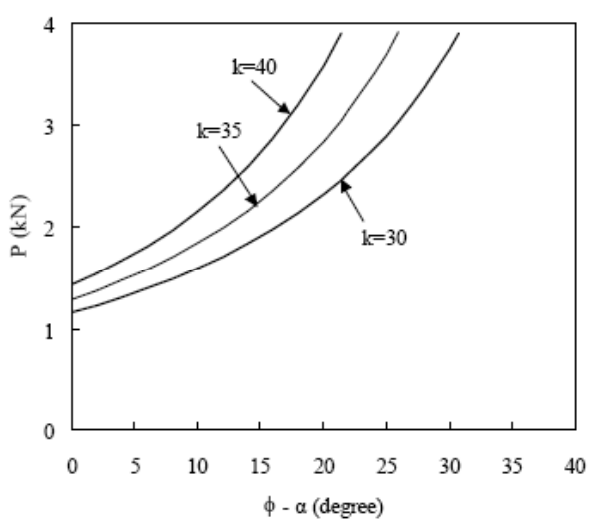

Fig. (9). Calculated critical cutting force, $P$.

Equation (8) is satisfied at the right tips of the 3 curves in the figure. The vertical axis is $P$, the lateral force component. $P$ at the right tip of the 3 curves is $3.9 \mathrm{kN}$. These data represent a cutting depth of $1 \mathrm{~cm}$ and a depth into the figure of 1 $\mathrm{cm}$ (Fig. 2). The forces on an actual disc cutter are expected to be several times as large as the forces indicated above.

The resultant force $p_{\mathrm{A}}$ on point $\mathrm{A}$ in Fig. (2) is believed to have the greatest effect on wear and is estimated using the following equation [7]:

$$
p_{A}=-(n+1) \frac{F}{t / \sin \theta}
$$

The computation results are shown in Fig. (10). The right tips of the curves represent cases where Eq. (8) is exactly satisfied. The highest resultant force in the figure occurs when $k=30^{\circ}$ at approximately $140 \mathrm{MPa}$. The shear strength is assumed to be $40 \mathrm{MPa}$, so this is 3.7 times the shear strength. If one assumes that the uniaxial compressive strength is 3 times the shear strength, $p_{\mathrm{A}}$ is approximately 1.2 times this uniaxial compressive strength. Roxborough and Phillips [11] assumed that the vertical stress on the rock di- 
rectly under the disc cutter was equal to the uniaxial compressive strength; the results here are not far different from their findings. The computation results in Fig. (10) are proportional to the shear strength and the cutting depth, so values under strengths and cutting depths other than those assumed in the present calculations can easily be calculated.

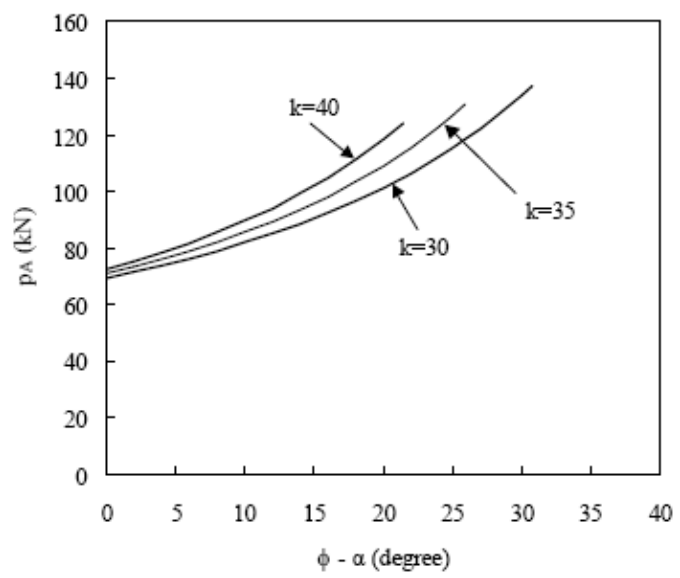

Fig. (10). Maximum resultant stress, $p_{\mathrm{A}}$.

\section{(Maximum Debris length $L$ )}

In addition to the issue of the TBM debris shape, inclusion of excessively large particles can block the loading and transport of the debris. Therefore, the authors also observed the distribution of the maximum debris length in the obtained data. As shown in Table 1, fragments weighing more than $20 \mathrm{~kg}$ were gathered at 9 locations. After calculating the maximum values for $L, L_{\max }$, the mean value of $L_{\max }, m$, and its standard deviation $S_{\mathrm{D}}$ were found; with $m \approx 15.2 \mathrm{~cm}$ and $S_{\mathrm{D}} \approx 5.3 \mathrm{~cm}$. Below, to simplify this investigation, the cumulative probability of $L_{\max }, F\left(L_{\max }\right)$, is assumed to be described by the Gumbel distribution [16], expressed as:

$F\left(L_{\max }\right)=\exp \left[{ }^{-} \exp \left(\left(\mu^{-} L_{\max }\right) / \beta\right)\right]$

$\beta$ is a parameter expressing the scatter in $L_{\max }$, and is found with the following[16]:

$\beta=\frac{\sqrt{6}}{\pi} S_{D}=4.13$

$\mu$, a parameter involved with the mean of $L_{\max }$, is found with the following[16]:

$\mu=m-0.577 \beta=12.82$

where 0.577 is the Euler-Mascheroni constant.

The two parameters are substituted into Eq. (11) and calculated and the results are shown in Fig. (11). To make the figure easier to understand, the vertical axis has been transformed into excavated volume, assuming a density of 2,500 $\mathrm{kg} / \mathrm{m}^{3}$. The largest $L_{\max }$ in all of the present measurements of debris was $26.7 \mathrm{~cm}$. The results shown in Fig. (11) are a kind of extrapolation, but careful consideration of this figure will reveal that, for example, a fragment measuring $L_{\max }=40$ $\mathrm{cm}$ will occur at intervals of volume about $6 \mathrm{~m}^{3}$, which is fairly often. Fragments with $L_{\max }=60 \mathrm{~cm}$ are only predicted at intervals of around $730 \mathrm{~m}^{3}$, and those with $L_{\max }=80 \mathrm{~cm}$, once in a volume of $93,000 \mathrm{~m}^{3}$, making them extremely rare. Those with $L_{\max }=100 \mathrm{~cm}$ are predicted to occur only once in $12,000,000 \mathrm{~m}^{3}$, which amounts to "never" in actual operations.

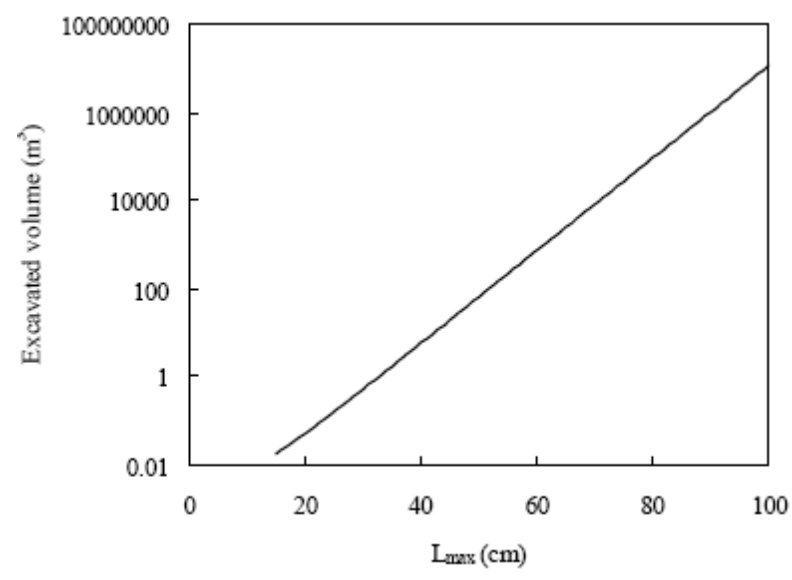

Fig. (11). Increase of the expected maximum length of debris, $L_{\max }$, with excavated volume.

Similar arguments based on the statistics of extremes have been employed in a number of fields, notably for predicting the speeds of typhoons and the scales of earthquakes $[17,18]$. These mathematics have almost never been applied to the study of TBM debris length, so the authors have attempted this very simplified analysis. The results do not conflict with other knowledge that the authors have come across to date, but it goes without saying that further investigation is needed.

\section{(Comparison with other Tunnels)}

Fig. (12) shows of the relationship between the numbers of disc cutters $N$ varied with the excavation diameter $D$ in 12 tunnels including Takisato Tunnel [19]. As can be seen, a good correlation was found between the two parameters, expressed in the following equation:

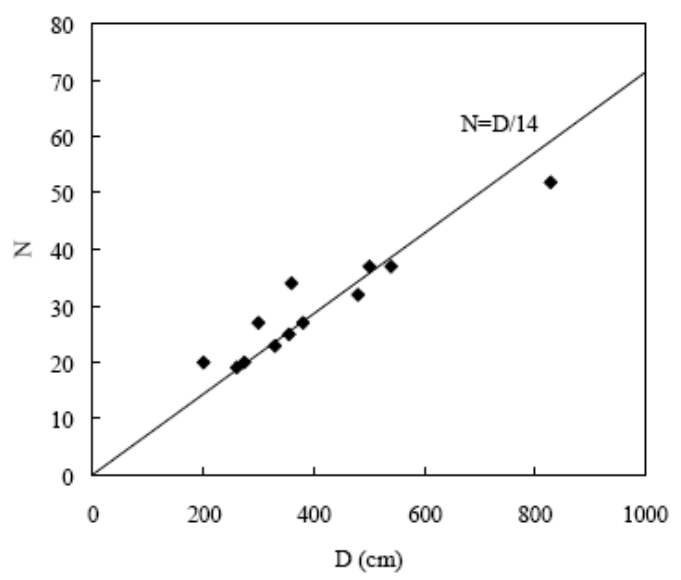

Fig. (12). Number of disc cutters, $N$, and excavation diameter, $D$. Data point for the Takisato tunnel is located at the far right $(D=830 \mathrm{~cm})$.

$N=D / 14$

The distance between adjacent grooves (distance between disc cutters) was calculated to be $7 \mathrm{~cm}$ using $s=0.5 \mathrm{D} / \mathrm{N}$. 
This was $8 \mathrm{~cm}$ in Takisato Tunnel, the object of the present study, a somewhat greater distance than average. The number of cutters on TBM cutter head increased linearly with D and then the maximum thrust Tmax $[\mathrm{MN}]$ in the TBM also increased linearly with D:

$T_{\max }=0.017 \mathrm{D}$

The average thrust $T_{\text {ave }}$ during operation was less than half this maximum thrust:

$T_{\text {ave }}=0.008 D$

In order to obtain a constant excavation speed that has no dependence on excavation diameter, the thrust must be proportional to the excavation area. However, this seems to be an impossible demand, projecting from current TBM design methods.

As we explained in a previous report [15], the findings in the domestic market indicate a tendency to suppress average thrust, and as a result, the cutting depth is quite low at $0.2-$ $1 \mathrm{~cm}$. Takisato Tunnel, the main tunnel of interest in this report, had the highest cutting depth of $1 \mathrm{~cm}$. This was a case where the cutting depth intended at the time of TBM design was actually accomplished during operation.

The ratio $s / t$ is a key parameter in considerations of the coupling of grooves with neighboring grooves. As shown in Fig. (13), $s / t$ tends to increase with strength of the rock mass. No correlation was found between $s / t$ and excavation diameter or disc cutter diameter. As can be seen in Fig. (13), $s / t$ takes on a fairly large value. It was only possible for coupling to take place during a single pass (a single rotation of the cutter head) in 3 of the tunnels, where $s / t$ had values of 9 or below. In the other tunnels, the cutting depth increased with every rotation of the cutter head, and at some point, coupling would finally occur with a neighboring groove (multipass). Coupling is the same whether it occurs in a single pass or after multiple passes, so it seems possible to make the approximation of debris shape and dimensions proposed here.

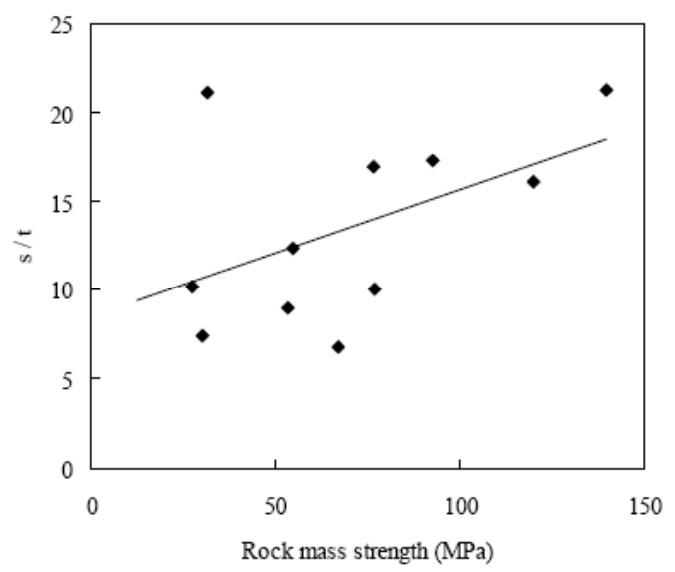

Fig. (13). Distance between adjacent grooves, $s$, normalized by cutting depth, $t$, against rock mass strength.

\section{CONCLUSIONS}

In addition to issues during loading and transportation, the dimensions and shapes of debris from a TBM have become important considerations during planning for its re-use.
It has appeared difficult to approach this topic theoretically, however, and it has almost never been investigated so far. This study proposes a method utilizing Nishimatsu's equation as an initial step in such a theoretical investigation. Nishimatsu's equation and its predecessor Merchant's equation were developed on the basis of an assumption that was quite bold in some senses. Researchers who use Nishimatsu's equation must recognize its basis and bear its limitations in mind while applying it, but it remains one of the methods with the most successful applications to date.

The most important parameter controlling the size of debris is the angle $\theta$ between the horizontal axis and the shear plane AB. Following Nishimatsu's approach, this value was assumed to be given by Eq. (4). The internal friction angle $k$, rock/tool friction angle $\phi$ and rake angle $\alpha$ determined by the shape of the cutting tool, are all included in Eq. (4). $\theta$ depends on values related to the rock, the rock/tool interface and the tool; Eq. (4) can be considered the most simplified possible expression for this functional relationship. It is possible that this equation will need to be revised based on future investigations, but we believe that Eq. (4) furnishes a valid expression as a starting point for further investigation.

The advantage of disc cutters is that one can rely on the occurrence of coupling between adjacent grooves. This generally means that the cutters require less specific energy than other excavation methods. This study developed Eq. (8) on the basis of Nishimatsu's equation to express the conditions under which coupling will occur. The value of the right side of Eq. (8) is determined by $k, \phi$ and $\alpha$ in the same way as $\theta$. Equation (8) can be considered the key point of both Nishimatsu's and this study's assertions. As mentioned above about $\theta$, it is likely that this expression will be revised and refined as more data are made available in the future, but Eq. (8) seems to furnish a valid starting point for further investigation.

The results of Eq. (8) were compared with the shapes and dimensions of debris collected in-situ. Of course, the collected debris passed through the process of loading and transport to the disposal site, so was also subject to secondary fracture. Therefore, maximum limits were obtained from Eq. (8) for both the dimension and length/thickness. We examined the results according to these standards and concluded that there was no contradiction between the predicted results and the observed results.

One of the advantages in using Nishimatsu's equation is that it allows estimation of the forces on the disc cutter and their distributions. Therefore, we calculated the maximum resultant force on the cutting edge of a disc cutter, which is quite closely related to the friction and wear there. The calculation results were within the range of plausibility. Nishimatsu's equation is one of a few which allows the researcher to state the distribution of force or stress on the rake face. This topic must be studied in more detail.

We performed a very simple analysis of the maximum values for debris length using the statistics of extremes. There were no data furnishing a basis for this, so it is difficult to evaluate these estimated results, but they are furnished as a suggestion in regard to this topic, which is a field that has received little attention. 
Finally, we performed a simple comparison and analysis of 12 tunnels in Japan in order to furnish some context for this investigation of Takisato Tunnel. The distance between adjacent grooves in Takisato Tunnel was somewhat greater than average, but the difference was only slight. The cutting depth was considerably greater than average, however. When the cutting depth is shallow, no coupling is caused with the adjacent groove during the first pass; the disc cutter must make multiple passes until it reaches a certain depth where coupling occurs. This "multi-pass mode" excavation is not desirable from the viewpoint of energy efficiency, but coupling is achieved eventually, and the argument pursued in this report appears to be an approximation of the excavation process.

This study left many topics to be covered in future investigations, but in a break from the scarcity of investigating the dimensions and shape of TBM debris, we believe it has pointed in one of the directions for investigation of these matters.

\section{ACKNOWLEDGEMENT}

The authors owe a great debt of thanks to many people who gathered debris for this course of research. We express our deepest appreciation to Taisei Corporation for their constant and generous cooperation.

\section{REFERENCES}

[1] S. Okubo, W. Chen, and K. Fukui, "A consideration on particle size distribution of fragments and energy required in uniaxial compression tests", J. Min. Metal. Inst. Japan, vol. 118, pp. 729-736, 2002. (in Japanese)

[2] K. Fukui, W. Chen, S. Okubo, and S. Sarata, "Particle distribution of cuttings in tunnel excavation", J. Min. Metal. Inst. Japan, vol. 119 , pp. 640-646, 2003. (in Japanese)

[3] L. Gertsch, A. Fjeld, B. Nilsen, and R. Gertsch, "Use of TBM muck as construction material", Tunnel. Underground Space Technol., vol. 15, pp. 379-402, 2000.
[4] K. Fukui, S. Okubo, T. Yoshino, and I. Kosaki, "Utilization of tunnel mucks as concrete aggregate", J. Min. Metal. Inst. Japan, vol. 120, pp. 380-387, 2004 (in Japanese).

[5] H. Tuncdemir, N. Bilgin, H. Copur, and C. Balci, " Control of rock cutting efficiency by muck size ", Int. J. Rock Mech. Min. Sci., Vol. 45, pp. 278-288, 2008.

[6] J. Jonak, and J. Podgorski, "Mathematical model and results of rock cutting modeling", J. Mining Sci., vol. 37, pp. 615-618, 2001.

[7] Y. Nishimatsu, "The mechanics of rock cutting", Int. J. Rock Mech. Min. Sci., vol. 9, pp. 261-270, 1972.

[8] G. Boothroyd, Fundamentals of Metal Machining and Machine Tools, McGraw-Hill Kogakusya (Tokyo), pp. 61-91, 1975.

[9] N. Takenaka, Kikai-Seisaku-Hou, Corona, Tokyo, 1968, pp. 12-16, (in Japanese).

[10] K. Nakayama, Sessaku-Kako-Ron, Corona, Tokyo, 1978, pp. $94-$ 120, (in Japanese).

[11] F. Roxborough, and H. R. Phillips, "Rock excavation by disc cutter", Int. J. Rock Mech. Min. Sci. Geomech. Abstr., vol. 12, pp. 361-366, 1975.

[12] R. A. Snowdon, M. D. Ryley, and J. Temporal, "A study of disc cutting in selected British rocks", Int. J. Rock Mech. Min. Sci. Geomech. Abstr., vol. 19, pp. 107-121, 1982.

[13] U. Yamaguchi, and Y. Nishimatsu, Introduction of Rock Mechanics, Tokyo Daigaku Shuppankai, 1991, p.181, (in Japanese).

[14] P. F. Rad, " Bluntness and wear of rolling disc cutters", Int. J. Rock Mech. Min. Sci. Geomech. Abstr., Vol. 12, pp. 93-99, 1975.

[15] S. Okubo, K. Fukui, and W. Chen, "Expert system for applicability of tunnel boring machines in Japan", Rock Mech. Rock Engng., vol. 36, pp. 305-322, 2003.

[16] E. J. Gumbel (T. Kawata, S. Iwai and S. Kase, Transl.), KyokuchiToukeigaku, Seisan Gijutsu Center Shinsya, Tokyo, §5 \& §6, 1978. (in Japanese)

[17] S. C. Chapman, G. Rowlands, and N. W. Watkins, "Extremum statistics: A framework for data analysis", Nonlinear Processes Geophys., vol. 9, pp. 409-418, 2002.

[18] H. W. Brink, and J. D. Konnen, "The statistical distribution of meteorological outliers", Geophys. Res. Lett., vol. 35, L23702, 2008.

[19] K. Fukui, S. Okubo, and K. Oda, "Relation between specifications and cutting forces of TBM", J. Min. Metall. Inst. Japan, vol. 118 , pp. 392-398, 2002 in Japanese. 